\title{
Rotational Vertebrobasilar Insufficiency Provoked By Distorted Trajectory of Subclavian Artery
}

\author{
Hasan Sumdani ${ }^{1 *}$, Zanab Shahbuddin ${ }^{2}$, Philip Church ${ }^{3}$ \\ ${ }^{1}$ Texas AEM University College of Medicine, Texas, USA; ${ }^{2}$ Seton Medical Center Austin, 1201 W 38th St, Austin, Texas, USA; \\ ${ }^{3}$ Department of Vascular Surgery, Seton Medical Center Austin, 1201 W 38th St, Austin, Texas, USA
}

\begin{abstract}
Rotational vertebrobasilar insufficiency (RVBI) is a rare form of decreased posterior brain circulation perfusion and can cause symptoms such as dizziness and vertigo. It is typically caused by unilateral dynamic narrowing of the vertebral artery during rotation of the head. Here we present the case of a 70-year-old male who experienced visual disturbances and syncopal episodes with rotation of his head to the left. Investigation was carried out with fluoroscopic imaging, and his symptoms were treated with vascular surgery. Later we discuss the distinctive characteristics of his imaging and his history which includes distorted vascular architecture and past surgery. The etiology of his RVBI has not been seen in the literature before.
\end{abstract}

Keywords: Rotational vertebrobasilar insufficiency; Bow hunter syndrome; Hemodynamic; Subclavian-carotid bypass; Vascular surgery; Case report

\section{INTRODUCTION}

Vertebrobasilar insufficiency $(\mathrm{VBI})$ is an uncommon cause of posterior circulation symptoms such as dizziness, vertigo, loss of balance or coordination, syncope, nausea, vomiting, visual disturbances, unilateral changes, and more. A rare form of VBI is rotational vertebrobasilar insufficiency (RVBI) which can produce the same symptoms, however, the cause is due to rotating the head [1-3].

RVBI is also known as Bow Hunter's Syndrome and is most classically described as being caused by compression of the vertebral artery by the bony elements of the cervical spine at the atlantoaxial region during rotation of the head to the contralateral side [4]. Many cases are comorbid with atherosclerotic, stenotic, or otherwise compromised vascular anatomy [4]. Rare cases of RVBI have been associated with other pathological mechanisms or anatomy such as bilateral rotation of the head, tilting the head, presence of distal pseudoaneurysm, osteophytes at lower cervical regions, and neck muscle hypertrophy $[2,4,5]$. Treatments are usually centered around conservative medical management with anticoagulation agents and education or surgical fusion of the cervical spine [4].

\section{CASE REPORT}

A 70-year-old male presented to his provider with complaints of paresthesias in the left arm, blurred vision, drop attacks, and syncopal episodes that were reproduced with rotation of his head to the left. He also experienced shortness of breath with left side head rotation. Symptoms were relieved with return to neutral positioning and did not occur unless he rotated his head to the left. His history was significant for hypertension, sleep apnea, atherosclerosis, coronary artery bypass grafting, sternum removal from post-operative infection, and coronary artery stenting.

\section{PRE-OPERATIVE STUDIES}

Patient underwent a diagnostic cerebral angiogram which was revealing of tandem basilar artery stenoses with the proximal defect approximately $56 \%$ narrowed and the distal defect approximately $82 \%$ narrowed. There was also occlusion of the basilar artery proximal to the superior cerebellar arteries' branching points (Figure 1).

The left subclavian artery was then examined and was shown to have a severe kinking along its trajectory before the vertebral artery branch which decreased its natural diameter to approximately $50 \%$ with the neck resting in the neutral position (Figure 2). This stenosis exhibited dynamic narrowing with rotation of the head to the left, exacerbating the stenosis to approximately $75 \%$.

Catheterization of the vascular system on the right side was unremarkable (Figure 3A), however, after comparison with the

Correspondence to: Hasan Sumdani, Texas A\&M University College of Medicine, Texas, USA, Tel: 817-914-4984; E-mail: hsumdani@medicine.tamhsc.edu Received: February 25, 2019; Accepted: March 28, 2019; Published: April 04, 2019

Citation: Sumdani H, Shahbuddin Z, Church P (2019) Rotational Vertebrobasilar Insufficiency Provoked By Distorted Trajectory of Subclavian Artery. J Vasc Med Surg 7: 2. doi: 10.35248/2329-6925.19.7.381

Copyright: (C) 2019 Sumdani H, et al. This is an open-access article distributed under the terms of the Creative Commons Attribution License, which permits unrestricted use, distribution, and reproduction in any medium, provided the original author and source are credited. 
right side, the left vertebral artery was noted to be dominant. Examination of the left vertebral artery was not revealing of any fixed or dynamic narrowing (Figure $3 \mathrm{~B}$ and $\mathrm{C}$ ). The studies made it clear that a subclavian-carotid bypass procedure could be most beneficial with regard to benefits and risks.

\section{OPERATIVE REPORT AND POST-OPERATIVE COURSE}

After consent was obtained and general anesthesia undertaken,

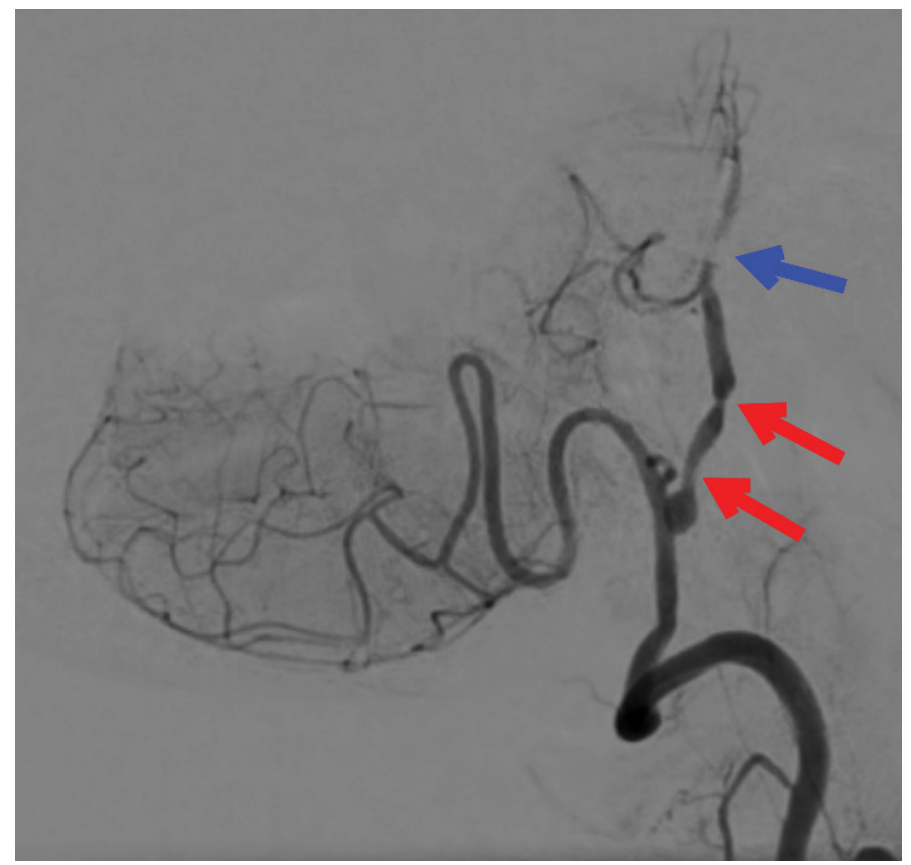

Figure 1: Left vertebral artery angiogram. A lateral view of the posterior brain circulation shows the vertebral artery at the bottom right with the basilar artery branching off. Two stenoses (red arrows) in series are seen through the course of the basilar artery with the distal lesion being worse than the proximal. The occlusion (blue arrow) is seen as a filling defect distal to the stenoses. the subclavian-carotid bypass procedure was initiated with a 6 centimeter $(\mathrm{cm})$ incision beginning at the lateral border of the left sternocleidomastoid approximately $1 \mathrm{~cm}$ above and parallel to the clavicle and extending laterally. To expose the common carotid artery, structures such as the scalene fat pad and lymphatic vessels were reflected, and the internal jugular vein was retracted. Upon exposure, the common carotid artery was dissected out for a length of $4 \mathrm{~cm}$.

To facilitate exposure of the subclavian artery, the anterior scalene muscle was cut at its inferior border and reflected superiorly. After protection of structures such as the phrenic nerve and brachial plexus, the subclavian artery was identified and dissected out between the two landmarks.

After administration of heparin, the common carotid was clamped and underwent $1 \mathrm{~cm}$ arteriotomy on its lateral aspect. A 7 millimeter $(\mathrm{mm})$ graft was sutured to the arteriotomy site and positioned superficial to the phrenic nerve to reach the subclavian artery which underwent arteriotomy on the anterior aspect for endto-side anastomosis.

Pulse was felt along both arteries and the connecting graft. Doppler testing revealed brisk and regular signaling. Heparin was then reversed with protamine, and the wound was closed in corresponding layers. There was minimal blood loss and no complications.

The patient's functional status after surgery was normal and notable for restoration of ability to rotate head to the left without any neurological or other sequelae of decreased perfusion to the posterior circulation.

\section{DISCUSSION}

One unique consideration about this case is that the patient's mechanism of RVBI may be due to the abrupt trajectory change of his subclavian artery, causing the brain to be more vulnerable to perfusion fluctuations in the vertebral artery by the bony elements
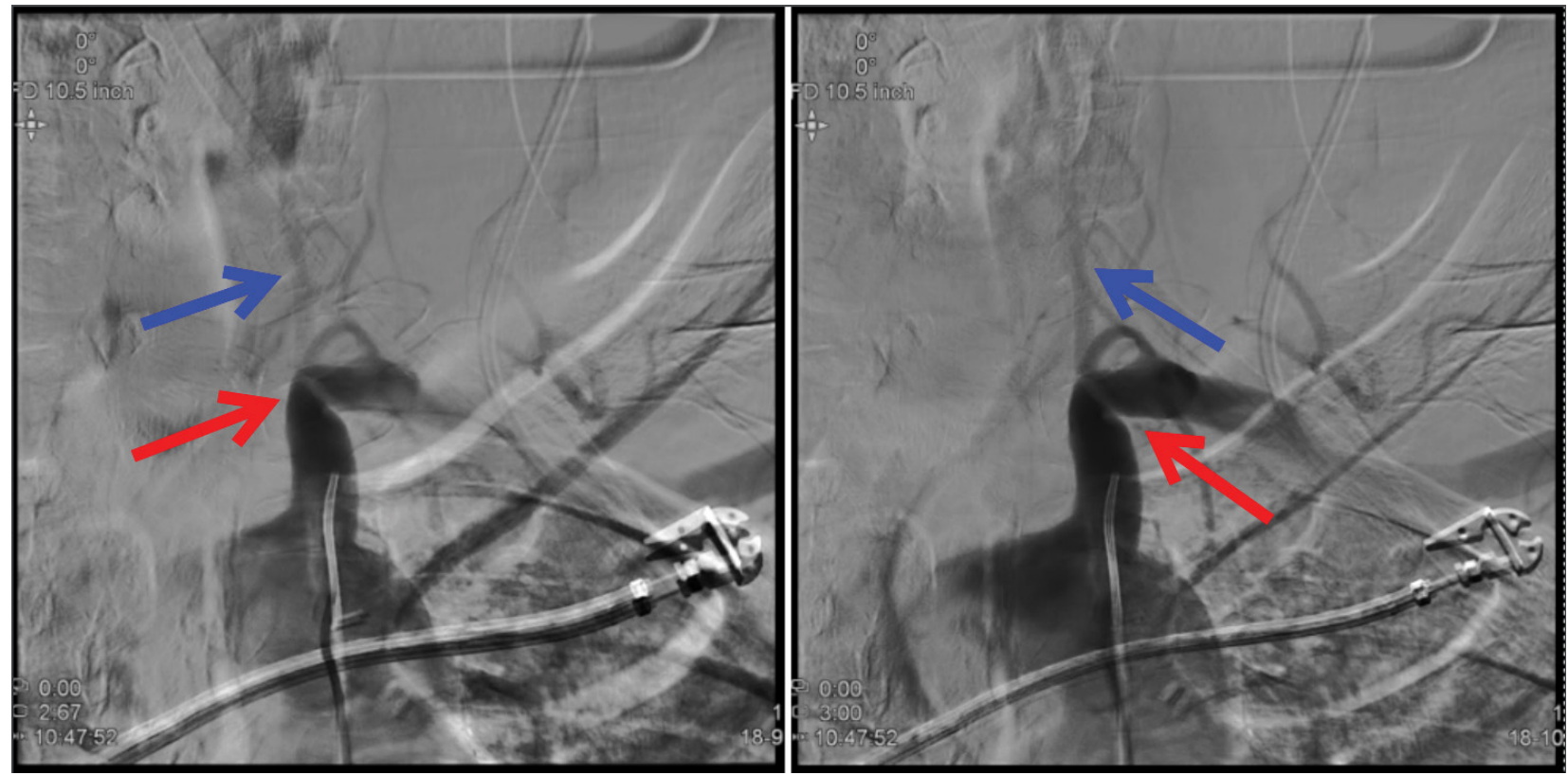

Figure 2: Left subclavian artery angiogram. Two anterior-posterior images of the subclavian artery and its branches are shown with contrast injected. The subclavian artery is shown coming off the aorta and is labeled by the red arrow. The vertebral artery is shown coming off the subclavian artery and is labeled by the blue arrow. 

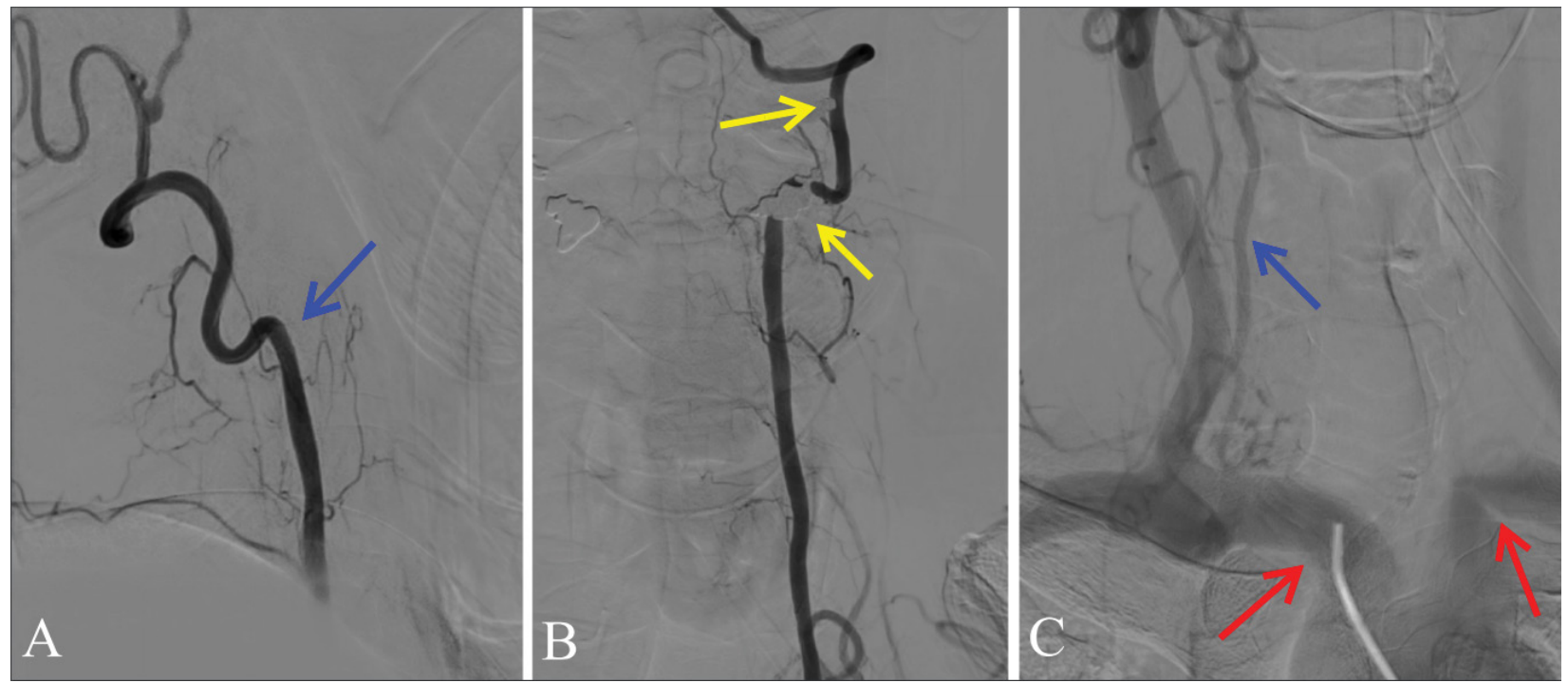

Figure 3: Normal anatomy of right vertebral artery, left vertebral artery, and right subclavian artery. Figure 3A shows the right vertebral artery (blue arrow) from the lateral view without any obvious narrowing lesions or other filling defects. Figure 3B shows the left vertebral artery in an anterior-posterior view. Yellow arrows depict artifact from patient's teeth that should not be mistaken for filling defects. Figure 3C compares right and left subclavian arteries (red arrows), and shows a right vertebral artery (blue arrow) without any compromising lesions.

of the cervical spine. This is supported by the dynamic narrowing of the subclavian artery that occurs when he turns his head to the left. Another source of evidence is the presence of paresthesias in his left upper extremity which would have been puzzling if his RVBI was due to vertebral or other distal artery pathology only.

The stenoses in his basilar artery are likely to have been contributing factors for the syncopal episodes that the patient experienced. It would be interesting to have observed if the patient would have suffered from RVBI if he did not have these stenoses.

The patient's history of coronary artery bypass grafting and removal of his sternum due to infection may have been other provocative factors in the development of his Bow Hunter's Syndrome. Though his heart surgery occurred over 10 years prior to his presentation with syncopal episodes, it is posited that the patient's left subclavian artery may have developed the anomalous kinking and stenosis because of the bypass surgery and the removal of his sternum that followed shortly after.

Though the classic case of Bow Hunter's Syndrome presents with symptoms from rotation of the head to the contralateral side, the patient suffered from syncope and more when turning his head to the ipsilateral side. This is likely explained by the kinking left subclavian artery as well since there was no dynamic narrowing of either vertebral arteries during head rotation. This feature of his condition helped in deciding the best treatment modality, too, since a cervical spine procedure, the usual treatment for RVBI, likely wouldn't have affected his symptoms as positively as the subclavian-carotid bypass that was performed instead. His case is made interesting still in that he also did not suffer from some commonly reported symptoms such as dizziness, vertigo, nausea, vomiting.

\section{CONCLUSION}

This report describes the case of a 70-year-old male with rotational vertebrobasilar insufficiency secondary to anomalous subclavian artery kinking. The patient's symptoms completely resolved following subclavian-carotid bypass surgery. The etiology of this man's Bow Hunter's Syndrome has not been seen in the literature before.

\section{REFERENCES}

1. Zaidi HA, Albuquerque FC, Chowdry SA, Zabramski JM, Ducruet AF. Diagnosis and management of bow hunter's syndrome: 15 year experience at Barrow Neurological Institute. World Neurosurgery. 2014;82(5):733-738.

2. Sarkar J, Wolfe SQ, Ching BH, Kellicut DC. Bow hunter's syndrome causing vertebrobasilar insufficiency in a young man with neck muscle hypertrophy. Ann Vasc Surg. 2014;28: 1032. e1-1032.e10.

3. Buch VP, Madsen PJ, Vaughan KA, Koch PF, Kung DK. Rotational vertebrobasilar insufficiency due to compression of a persistent first intersegmental vertebral artery variant: case report. J Neurosurgery: Spine. 2017;26(2):199-202.

4. Choi KD, Choi JH, Kim JS, Kim HJ, Kim MJ. Rotational vertebral artery occlusion: mechanisms and long-term outcome. Stroke. 2013;44(7):1817-1824.

5. Hernandez RN, Wipplinger C, Navarro-Ramirez R, Patsalides A, Tsiouris AJ. Bow Hunter Syndrome with Associated Pseudoaneurysm. World Neurosurgery. 2019;122:53-57. 